\title{
Interferential seeing monitor
}

\author{
A. Habib, M. Sabil, Z. Benkhaldoun, and Y. El Azhari
}

\begin{abstract}
UFR: APHE, Université Cadi Ayyad, Faculté des Sciences Semlalia, Av. Prince My Abdellah, BP 2390 Marrakech, Morocco e-mail: a.habib@ucam.ac.ma
\end{abstract}

Received 6 November 2007 / Accepted 6 February 2008

\section{ABSTRACT}

\begin{abstract}
Context. This work is a part of the development of observational instruments for the study of optical turbulence in the atmosphere. These instruments are developed for the optimization of high angular resolution and adaptive optics techniques.

Aims. We present a new approach to estimate the astronomical seeing which is a fundamental parameter in high angular resolution, in adaptive optics and site testing. Based on this approach, we developed a simple low-cost seeing monitor, called Interferential Seeing Monitor (hereafter ISM).

Methods. The principle of the ISM is based on the study of the diffraction-interference pattern produced by a Young's double-slit in a telescope focus. From the shape of that pattern, we determine the phase difference between the diffracted light rays that meet on the image plane. Then, the phase structure function is calculated which leads to the seeing value.

Results. Two seeing measurement campaigns were carried out at Oukaimden observatory and the ENS de Marrakech site using this new seeing monitor and a DIMM monitor. The two results are in good agreement, which makes the ISM a reliable instrument.
\end{abstract}

Key words. atmospheric effects - methods: observational - techniques: interferometric - site testing

\section{Introduction}

When a plane wave passes through the atmosphere, wavefront deformations are generated by random variations of the air refractive index. This phenomenon is caused by atmospheric turbulence and reduces the angular resolution of ground-based telescopes. The knowledge of atmospheric turbulence parameters is essential in high angular resolution and adaptive optics where there is a need to correct the astronomical image distortions. The seeing $\varepsilon$ is one of the astronomical parameters used to characterize the effects of atmospheric turbulence on the telescope resolution. It is defined as the Full With at Half Maximum (FWHM) intensity of a point source image and is related to Fried's parameter $r_{0}$ by $\varepsilon=0.98 \lambda / r_{0}$. The importance of atmospheric parameters has led to the development of many instruments for astronomical observation in order to characterize the optical turbulence.

The Differential Image Motion Monitor (DIMM) is one such instrument. It consists of two sub-apertures of a small telescope that form two images of a star on a camera detector (Martin 1987; Sarazin \& Roddier 1990). This instrument allows us to estimate the seeing from the variance of the differential motion of the images. These images are obtained by using a mask with two holes where a prism is placed in one of them to form a second image on the camera detector. It is also possible to use a beam splitter to obtain two images of the same star (Sarazin \& Roddier 1990). The DIMM is now commonly used for the qualification of astronomical sites (Vernin \& Muñoz-Tuñón 1994).

In addition, many other techniques allow one to determine the seeing like the MASS (Multi-Aperture Scintillation Sensor) that consists of four concentric circular apertures of different radii. Four photomultipliers are used to calculate the scintillation indices (normal and differential) in each aperture. Thus, the turbulence intensity is measured in 6 fixed layers, or 3 floating layers, from 0.5 to $16 \mathrm{~km}$ with a vertical resolution of 0.5 of altitude (Tokovinin et al. 2003, 2007;
Kornilov et al. 2003, 2007). Hence, the seeing is deduced from the turbulence vertical profile.

The SCIDAR (SCintillation Detection And Ranging) is also an important turbulence profiler that allows us to obtain atmospheric parameter profiles (Rocca et al. 1974). It is based on the study of the cross-correlation function of double star scintillation. This study is conducted on an analysis plane that lies at a distance $h_{\mathrm{sg}}$ below the entrance pupil of the telescope . This makes it possible to detect turbulence in lower turbulent layers (Fuchs et al. 1998). The Single Star Scidar (Rocca et al. 1990; Habib et al. 2005, 2006) was developed in order to use single star scintillation for the determination of the optical turbulence profiles. Based on these profiles, one can calculate the seeing as well as all other parameters for adaptive optics.

The GSM (Generalized Seeing Monitor) is an instrument that allows us to estimate Fried's parameter $r_{0}$, the outer scale, the coherence time and the isoplanatic angle of atmospheric turbulence (Avila et al. 1997; Ziad et al. 2000). It measures the angle of arrival fluctuations by using four independent telescopes. Two of them are mounted on the same guiding mount. Fried's parameter is deduced from the differential variance while the other parameters are deduced from the spatial covariance and the spatiotemporal correlation of the angle of arrival.

In interferometry, the atmospheric turbulence causes attenuation of the long-exposure fringe visibility by a factor that depends on Fried's parameter $r_{0}$ (Fried 1966). Thus, it is possible to estimate $r_{0}$ by studying the shape of that visibility (Roddier \& Roddier 1978).

In 2007, Kellerer \& Tokovinin (Kellerer \& Tokovinin 2007a,b) introduced a new method, termed FADE (FAst DEfocus), to measure the atmospheric time constant which is related to the variance of the defocus speed. The variance of defocus, which is proportional to $r_{0}^{-5 / 3}$, gives a measure of the seeing. They have also derived a new formula for the structure function of the fringe phase (piston) in a long-baseline interferometer. 


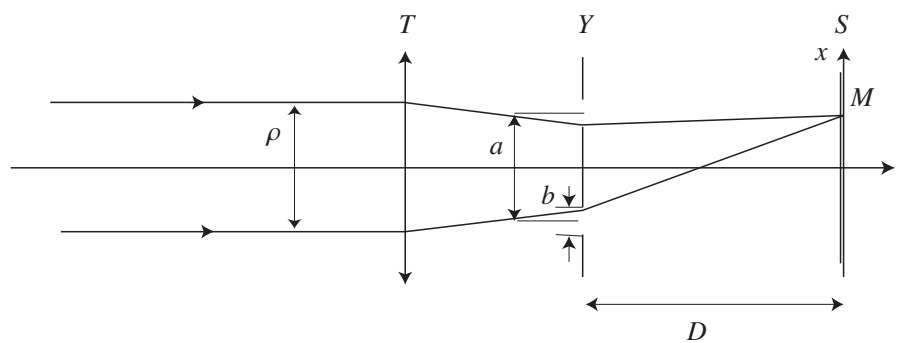

Fig. 1. Optical scheme of ISM monitor with (from left to right) the telescope (T) of focal length $f_{\mathrm{t}}$, Young's double-slit (Y) and a screen (S) or camera detector.

In this paper we present a new method for seeing determination that is based on the study of the diffraction pattern generated by a Young's double-slit in a telescope focus. Indeed, the shape of the instantaneous intensity, along the direction perpendicular to the two slits, depends on the phase difference between the two diffracted rays. This allows us to estimate the phase structure function which leads to the seeing estimate. This instrument will be called the Interferential Seeing Monitor (ISM).

\section{Principle of the ISM monitor}

\subsection{Theory}

The principle of the interferential seeing monitor is based on the study of the diffraction-interference pattern generated by the optical system shown in Fig. 1. This figure shows two light beams, separated by a distance $\rho$, that pass through the telescope (T), of focal length $f_{\mathrm{t}}$, then through the two Young' slits (Y) which are separated by a distance $a$. The two diffracted rays meet on a screen $(\mathrm{S})$ that is located at a distance $D$ from the double slit (in the telescope focus).

The instantaneous light intensity along the $x$-axis, which is perpendicular to the two slits, is given by (Born \& Wolf 1980)

$I(x)=\frac{I_{0}}{2} \operatorname{sinc}^{2}\left(\frac{\pi b}{\lambda} \frac{x}{D}\right)\left[1+V \cos \left(\frac{2 \pi a}{\lambda} \frac{x}{D}+\Delta \varphi_{\mathrm{t}}\right)\right]$

where $\Delta \varphi_{\mathrm{t}}$ is the phase difference between the two rays, introduced by atmospheric turbulence, $\lambda$ the wave length, $b$ the slit width, $I_{0}$ the total intensity, and $V$ the visibility factor.

Assuming that the turbulence is homogeneous and isotropic, this phase difference can be written, for a given position $r$, as

$\Delta \varphi_{\mathrm{t}}(\rho)=\varphi(r+\rho)-\varphi(r)$

where $\rho$ is the distance between the two parallel rays.

Figure 2 shows the shape of $I(x) / I_{0}$ for a given value of $\Delta \varphi_{\mathrm{t}}$ and a visibility $V=1$. As we can see, the phase difference $\Delta \varphi_{\mathrm{t}}$ causes a displacement of the intensity curve perpendicularly to the two slits.

Thus, from the shape of this curve, which depends on $\Delta \varphi_{\mathrm{t}}$, we can extract the instantaneous phase difference. To perform this, we calculate the following ratio

$R\left(\Delta \varphi_{\mathrm{t}}\right)=\frac{\int_{x_{1}}^{x_{2}} I(x) \mathrm{d} x-\int_{y_{2}}^{y_{1}} I(x) \mathrm{d} x}{\int_{y_{2}}^{x_{2}} I(x) \mathrm{d} x}$

where the integral bounds $y_{1}, y_{2}, x_{1}$ and $x_{2}$ are shown in Fig. 2.

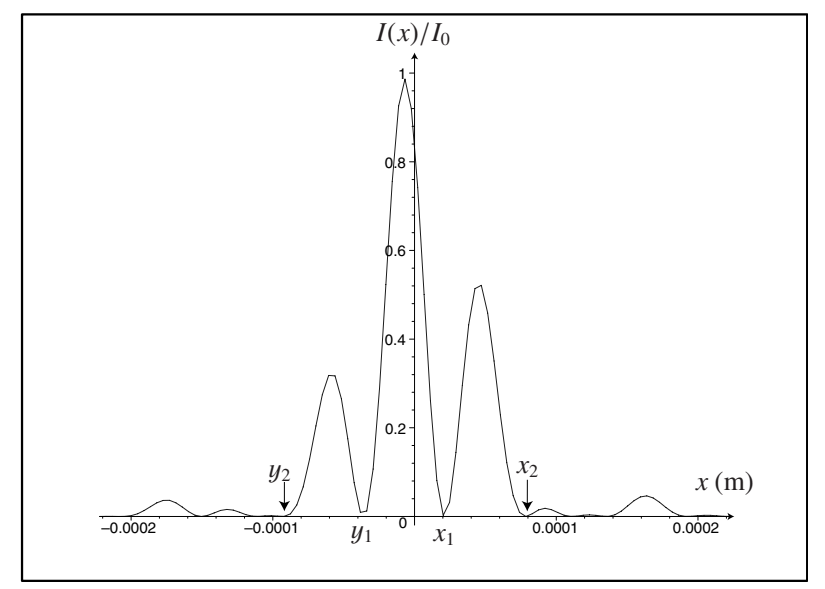

Fig. 2. Theoretical cut of $I(x) / I_{0}$ along the $x$-axis for a given value of $\Delta \varphi_{\mathrm{t}}$. The shape of the intensity depends on the phase difference. This curve was obtained by using the following configuration: $a=175 \mu \mathrm{m}$, $b=90 \mu \mathrm{m}, D=0.02 \mathrm{~m}, f_{\mathrm{t}}=2.8 \mathrm{~m}$ and $\lambda=0.5 \mu \mathrm{m}$.

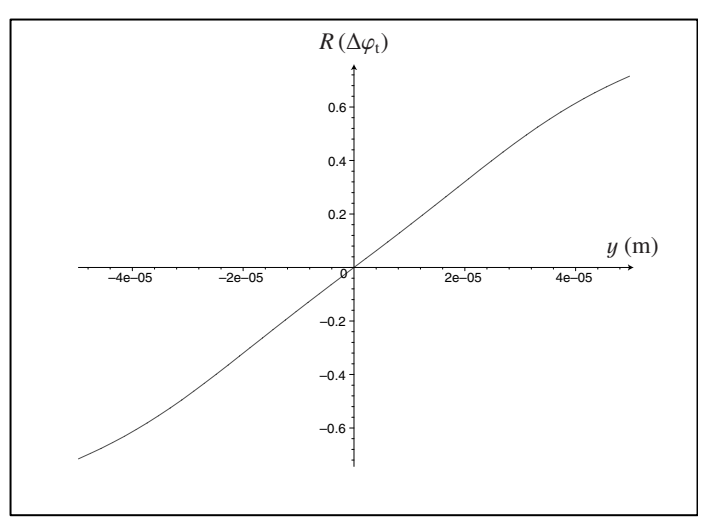

Fig. 3. The ratio $R$ as a function of $y=\frac{\lambda D}{2 \pi a} \Delta \varphi_{\mathrm{t}}$ obtained for the same configuration given in Fig. 2. From this simulation we have concluded that for the highest seeing value (5 or 6 arcsec), the maximal fringe displacement is $2 \times 10^{-5} \mathrm{~m}$ and the slope of linear proportion is $S=15830 \mathrm{~m}^{-1}$.

This ratio, which is not sensitive to the total intensity $I_{0}$ and erratic vibrations of the telescope, is represented as a function of fringe displacement $y=\frac{\lambda D}{2 \pi a} \Delta \varphi_{\mathrm{t}}$ in Fig. 3. This figure was obtained by a simulation programme for: $a=175 \mu \mathrm{m}, b=90 \mu \mathrm{m}$, $D=0.02 \mathrm{~m}, f_{\mathrm{t}}=2.8 \mathrm{~m}$ and $\lambda=0.5 \mu \mathrm{m}$.

From simulation results, we conclude that the fringe displacement does not exceed $2 \times 10^{-5} \mathrm{~m}$ even for a high seeing value like 5 or 6 arcesc. Consequently, the ratio $R\left(\Delta \varphi_{\mathrm{t}}\right)$ is a linear function of $y\left(-2 \times 10^{-5} \mathrm{~m}<y<2 \times 10^{-5} \mathrm{~m}\right)$ and can be written as

$R\left(\Delta \varphi_{\mathrm{t}}\right)=S \frac{\lambda D}{2 \pi a} \Delta \varphi_{\mathrm{t}}$

where $S$ is the slope of the linear portion.

If we take into account the loss of fringe visibility $(V<1)$, the shape of diffraction pattern changes slightly but the ratio $R$ remains a linear function of $y$. To be able to measure the phase difference, the fringe displacement should not cause the disappearance of the lateral fringes, which correspond to a maximum seeing value of about 7 arcsec depending on the ISM configuration. Generally the maximum seeing value does not exceed 3 or 4 arcsec, which makes the Eq. (4) valid in most circumstances. 
Once $\Delta \varphi_{\mathrm{t}}$ is extracted, we can calculate the phase structure function that corresponds to the distance $\rho$

$D_{\varphi}(\rho)=\left[(\varphi(r+\rho)-\varphi(r))^{2}\right]=\left[\left(\Delta \varphi_{\mathrm{t}}(\rho)\right)^{2}\right]$.

Here the square brackets denote an average over a given series of short-exposure images. The atmospheric turbulence is supposed to be locally homogeneous and isotropic.

On the other hand, assuming the Obukhov low for atmospheric refractive index fluctuations, the phase structure function is given by (Roddier 1981)

$D_{\varphi}(\rho)=2,91\left(\frac{2 \pi}{\lambda}\right)^{2} \cos ^{-1}(\gamma) \rho^{5 / 3} \int_{0}^{+\infty} C_{n}^{2}(h) \mathrm{d} h$

where $\cos ^{-1}(\gamma)$ is the air mass, $\lambda$ the wavelength and $C_{n}^{2}(h)$ the air refractive index structure function which depends upon the altitude $h$. This equation is valid under a near field approximation.

Since Fried's parameter $r_{0}$ is given by (Roddier 1981)

$r_{0}=\left[0.423\left(\frac{2 \pi}{\lambda}\right)^{2} \cos ^{-1}(\gamma) \int_{0}^{+\infty} C_{n}^{2}(h) \mathrm{d} h\right]^{-3 / 5}$

and using the fact that

$\varepsilon=0.98 \frac{\lambda}{r_{0}}$

relations (6) and (7) allow us to obtain

$\varepsilon=0.308 \lambda \frac{D_{\varphi}^{3 / 5}(\rho)}{\rho}$.

This equation allows us to obtain the seeing from the phase structure function. The distance $\rho$ depends on the distance between the two slits and the optical magnification.

\subsection{Configuration of the ISM}

The ISM we used in our experiments consists of a small telescope (20 to $35 \mathrm{~cm}$ ), mounted on an equatorial mount, equipped with a Young's double-slit $(b=90 \mu \mathrm{m} ; a=175 \mu \mathrm{m})$ and a CCD camera. For the configuration given in Fig. 2, the projected size of the slits at the telescope entrance aperture is $12.6 \mathrm{~mm}$. We expect that the use of a mask with two $12.6 \mathrm{~mm}$ slits, over the entrance aperture of the telescope, could also lead to the same results. Since the integrals bounds $\left(x_{1}, x_{2}, \ldots\right)$ are proportional to $1 / a$, and $\operatorname{sinc}^{2}\left(\frac{\pi b}{\lambda} \frac{x}{D}\right)$ is zero at $x_{3}=\lambda D / b>x_{2}$, the two parameters $a$ and $b$ should be chosen smaller to obtain fringes that are as large as possible. If $x_{1}$ and $x_{2}$ are larger, the ratio $R$ will be calculated over a greater number of pixels, which reduces the noise error. However, if $b$ is too small the light flux becomes very weak which reduces the signal-to-noise ratio. Generally, only the three central fringes are visible (Fig. 4) which is enough to estimate the seeing. On the other hand, the exposure time should be small enough in order to freeze the turbulence and obtain an instantaneous image intensity. The camera detector was placed in the telescope focus in order to obtain a diffraction pattern around the geometrical single star image (Fraunhofer case). The camera was connected to a computer via the USB serial port.

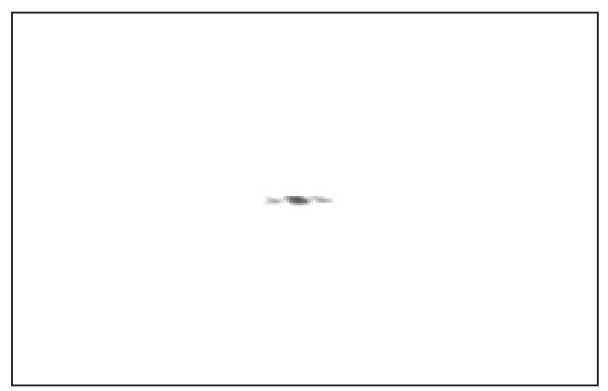

Fig. 4. Example of the diffraction pattern obtained with the ISM instrument at Oukaimden. The three central fringes are visible.

To calculate the integrals in Eq. (3) we start by the determination of the central fringe centroid and the integral bounds $x_{1}$, $x_{2}, y_{1}$ and $y_{2}$. Afterwards, we calculate the phase difference $\Delta \varphi_{\mathrm{t}}$ using Eq. (4). The phase structure function given by Eq. (5) is calculated by using a series of 200 or 300 short-exposure frames ( 5 to $10 \mathrm{~ms}$ ). Since the standard error of the phase structure function decreases as the square root of the number of independent samples, the number of frames should be large enough (a few hundred) to give a good estimate of that quantity.

The time resolution depends on the number of frames, the exposure time and the computer-camera features. For a series of 300 independent frames, the times resolution in our experiment was $20 \mathrm{~s}$.

In addition, to have an instantaneous diffraction pattern, the exposure time should be chosen sufficiently smaller than the turbulence evolution time and the wind crossing time of the turbulence over the aperture of the telescope. For a long exposure, the instantaneous intensity given by Eq. (1) becomes invalid and the seeing value is underestimated. Furthermore, the images should be non-saturated in order to obtain a good estimate of the previous integrals.

\subsection{Some features of the ISM}

Some important points of the ISM instrument and comparisons with DIMM are:

The ISM seeing does not depend on the telescope diameter while the DIMM uses a small telescope of a diameter that is two times greater than the sub-aperture diameter.

In the ISM experiment, the diffraction pattern is always well focused (Fraunhofer's case). If not, we should study the diffraction pattern in Fresnel's case. In the DIMM experiment, the consequence of the use of a prism is that the two spots cannot be simultaneously well focused, which biases the seeing.

The formulas used in the ISM do not involve any approximations, which leads to a good estimation of phase difference. The formulas used in DIMM's theory to compute seeing from image motion variance (Sarazin \& Roddier 1990) were derived from a crude approximation (the baseline should be larger than the aperture diameter) and imprecise coefficients (Tokovinin 2002).

The noise has less effect on the integrals in Eq. (3) than on the variance of differential image motion in a DIMM. Thus the ISM seeing is less biased by the noise than the DIMM seeing. Nevertheless, it is recommended to use a bright star in order to reduce the bias on the seeing.

The Fried parameter is converted to seeing for a wavelength of $0.5 \mu \mathrm{m}$ (Eq. (8)). From simulation results, we concluded that the product $S \times \lambda$ (Eq. (4)) is independent of the wavelength. For example, in the previous configuration $S \times \lambda=7.7 \times 10^{-3}$. 


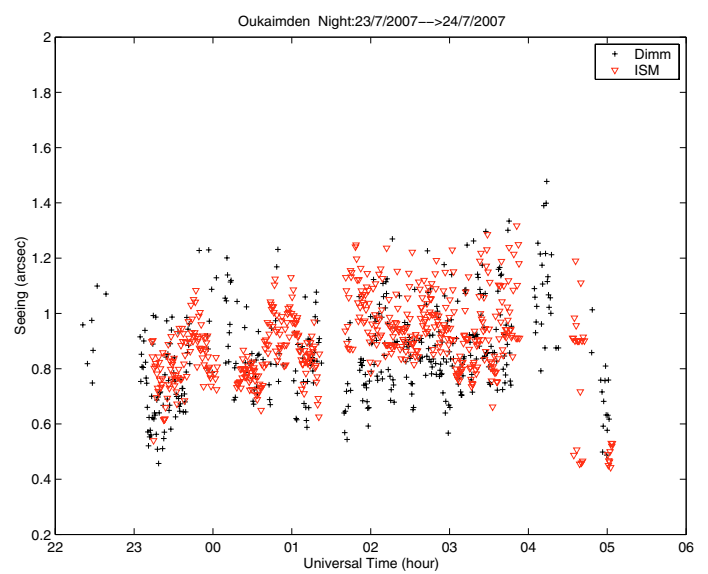

Fig. 5. Seeing as a function of universal time given by the ISM monitor (triangle) and the DIMM monitor (+) during the night of July 23, 2007 at Oukaimden Observatory.

Thus, for a given phase difference $\Delta \varphi_{\mathrm{t}}$ we obtain the same ratio $R\left(\Delta \varphi_{\mathrm{t}}\right)$ for different colored filters. In order to reduce the effect of the nonmonochromaticity of the light on ISM measurements, we should use a colored filter.

The ISM seeing can be affected by the nonuniformity of the irradiance over the two slits, that is why the diffraction pattern should be well centered around the geometrical image.

\section{Results}

Data reported here were recorded at the Observatoire Universitaire de l'Oukaimden $\left(7^{\circ} 52^{\prime} 52^{\prime \prime} \mathrm{W}, 31^{\circ} 12^{\prime} 32^{\prime \prime} \mathrm{N}\right.$; $2700 \mathrm{~m}$ above sea level) and ENS de Marrakech site $\left(7^{\circ} 32^{\prime} \mathrm{W}\right.$, $31^{\circ} 38^{\prime} \mathrm{N}$; $450 \mathrm{~m}$ above sea level), Marrakech, Morocco, during July and October 2007.

At Oukaimden we used, in the ISM experiment, a $35-\mathrm{cm}$ telescope, a Young's double slit (90 $\mu \mathrm{m}$ wide ; $a=175 \mu \mathrm{m})$ and a CCD camera. The phase structure function was calculated using a series of 300 images. The ISM instrument was installed inside the dome of the telescope while a DIMM monitor was installed outside (on the tower) very close to the telescope dome. The tower is about $12 \mathrm{~m}$ high in order to avoid the surface layer turbulence (Vernin \& Muñoz-Tuñón 1994). In the DIMM experiment, we used a 20 -cm telescope, a CCD camera and a mask with two sub-apertures placed at the entrance of the telescope. The diameter of each sub-aperture is $60 \mathrm{~mm}$ and the distance between them is $140 \mathrm{~mm}$. The exposure time in the two experiments was $10 \mathrm{~ms}$.

In Fig. 5 we presented the seeing as a function of universal time obtained during the night of July 23, 2007 where the observed star was Vega. As can be seen, the seeing measured by the two instruments (DIMM and ISM) is practically the same throughout the night.

Figure 6 shows a comparison between the histograms of the DIMM and ISM results. The average seeing given by the DIMM is 0.86 arcsec with a median value of 0.84 arcsec and that given by the ISM instrument is 0.89 arcsec with a median value of 0.9 arcsec. This value is close to the typical seeing value of 1.05 arcsec obtained at Oukaimden (Benkhaldoun et al. 2005). The slight averages difference of 0.03 arcsec could be related to the turbulence inside the telescope dome.

The correlation between the two results is shown in Fig. 7 where we presented the seeing given by the ISM monitor versus the seeing given by the DIMM monitor. The time sampling is
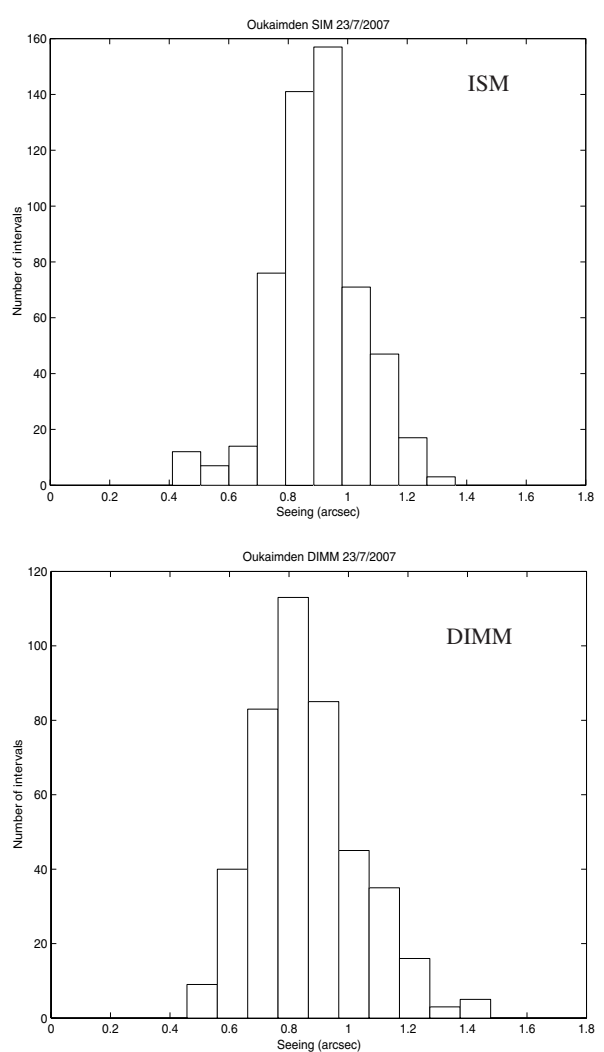

Fig. 6. Statistical distribution of seeing in the night of July 23, 2007 at Oukaimden Observatory.

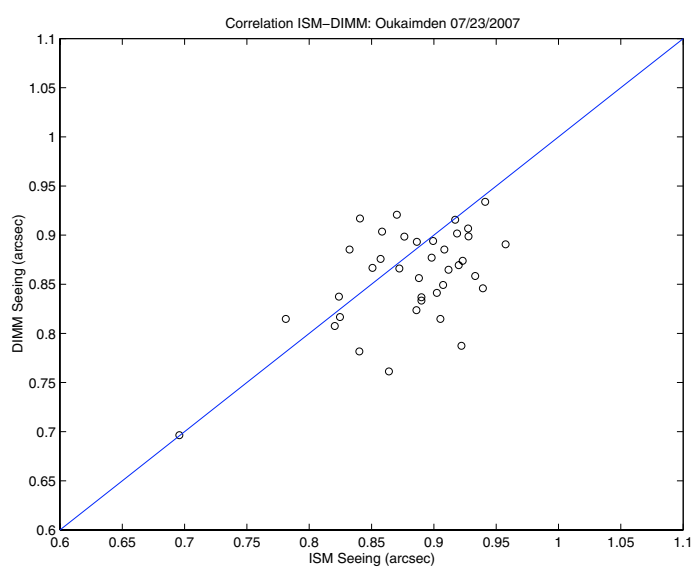

Fig. 7. Correlation between the seeing given by ISM monitor and that given by DIMM monitor. Each point represents an average seeing over 7 min.

$7 \mathrm{~min}$. This figure shows that the points are distributed around the straight line of slope one with a slight shift to the right because of the dome turbulence. This means that there is a good correlation between these results.

However, the scatter of the points could be related to the differences between the two instruments. Indeed, the DIMM seeing could be biased by the defocus of one of the two images (prism effect), the noise and the finite exposure time. The ISM uses an interferometric approach which leads to a precise measurement of the phase difference but it could be affected by the finite exposure time, the instrumental noise and nonmonochromaticity of the light. 

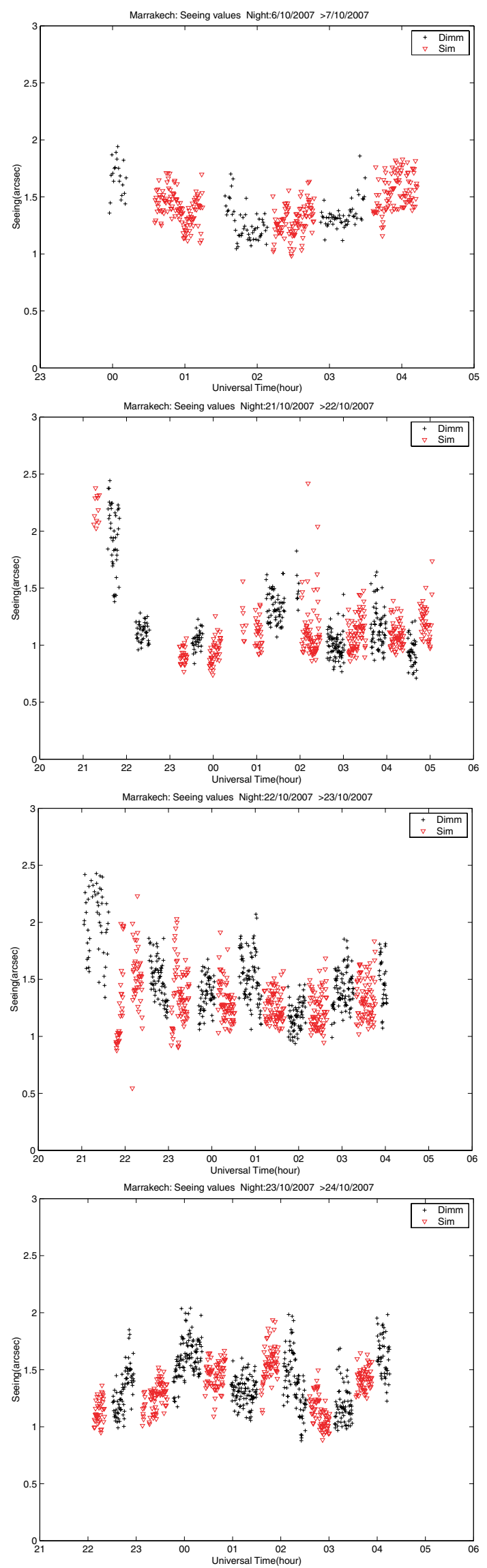

Fig. 8. Seeing as a function of time, given by the ISM monitor (triangle) and the DIMM monitor (+), during four nights at ENS de Marrakech site. The seeing evolution is the same.
Table 1. Statistical parameters of the seeing during the four nights at ENS-Marrakech site.

\begin{tabular}{ccccc}
\hline \hline & ISM & \multicolumn{3}{c}{ DIMM } \\
Night & $\langle\varepsilon\rangle$ & $\varepsilon_{\text {median }}$ & $\langle\varepsilon\rangle$ & $\varepsilon_{\text {median }}$ \\
\hline $10 / 06 / 2007$ & 1.45 & 1.47 & 1.36 & 1.32 \\
$10 / 21 / 2007$ & 1.10 & 1.05 & 1.20 & 1.10 \\
$10 / 22 / 2007$ & 1.41 & 1.37 & 1.48 & 1.43 \\
$10 / 23 / 2007$ & 1.40 & 1.42 & 1.41 & 1.42 \\
\hline
\end{tabular}

Another comparison between the ISM and the DIMM results was done at the ENS de Marrakech site during four nights. The meteorological conditions (low wind speed, low humidity,...) were favorable for good measurements. In this cross-calibration we used a $28-\mathrm{cm}$ telescope and interchanged the ISM and the DIMM on it. For both instruments, we used the same components (slits, mask, cameras) as in the Oukaimden campaign. The exposure time was $1 / 120 \mathrm{~ms}$ and the observed star was Capella.

Figure 8 shows the temporal evolution of the seeing during all nights and the statistical parameters are presented in Table 1. There is a good agreement between the two results. The seeing evolution is the same in both results during all nights, particularly in the night of 23 October where the seeing changed many times. Since we interchanged the two systems, on the same telescope, minor differences between the statistical parameters occurred because the data were not exactly contemporaneous.

This agreement between the results of ISM and DIMM instruments, which use different principles, confirms the validity of our approach. Thus, we conclude that the ISM monitor is a reliable instrument for seeing measurement.

\section{Conclusions}

A new instrument (ISM) for astronomical seeing determination is presented and validated. The principle of this instrument is based on the study of the diffraction pattern generated by a Young's double-slit in a telescope focus. This study consists of the extraction of the phase structure function from the instantaneous intensity in order to estimate the astronomical seeing. Comparison between the ISM results and the DIMM results allowed us to confirm the validity of our approach of seeing determination.

The time resolution is about 10 to $20 \mathrm{~s}$ depending on the computer's speed, the exposure time and the number of frames used to estimate the phase structure function.

The use of an interferometric method allows us to obtain a precise and fast measurement of the phase difference between two points, which leads to a good estimate of the astronomical seeing in real time.

Compared to the other techniques, the ISM seems to be the simplest instrument, easy to set-up, and low-cost. It requires only a Young's double-slit, a CCD camera (even a webcam) and a small telescope. Thus, it is an appropriate instrument for site testing.

An extended study of the effect of the defocus, the finite exposure time, the loss of visibility and the instrumental noise on the ISM is in progress. The results will be published in a forthcoming article.

Acknowledgements. This work has been performed in collaboration with Laboratoire d'optique et d'opto-élelctronique of ENS de Marrakech. Authors would like to thank all members of that laboratory for providing the site and equipment for the observation. Also, we want to acknowledge the staff of Oukaimden Observatory for their support. 


\section{References}

Avila, R., Vernin, J., \& Masciadri, E. 1997, 1ppl. Opt., 36, 7898

Benkhaldoun, Z., Abahamid, A., El Azhari, Y., \& Lazrak, M. 2005, A\&A, 441, 839

Born, M., \& Wolf, E. 1980, Principles Of Optics (Oxford: Pergamon Press)

Caccia, J.-L., \& Vernin, J. 1990, J. Geophys. Res., 95-D9, 683

Fried, D. L. 1966, J. Opt. Soc. Am. A, 55, 1427

Fuchs, A., Tallon, M., \& Vernin, J. 1998, Ast. Soc. of The Pac., 110, 86

Habib, A., Vernin, J., Benkhaldoun, Z., \& Lanteri, H. 2006, MNRAS, 368, 1456

Habib, A., Vernin, J., \& Benkhaldoun, Z. 2005, C.R. Physique 6

Kellerer, A., \& Tokovinin, A. 2007, A\&A, 461, 775

Kellerer, A., Sarazin, M., Butterley, T., \& Wilson, R. 2007, Appl. Opt., 46, 4754

Kornilov, V., Tokovinin, A., Vozyakova, O., et al. 2003, Proc. SPIE, 4839, 837
Kornilov, V., Tokovinin, A., Shatsky, N., et al. 2007, MNRAS, 382, 1268 Martin, H. M. 1987, PASP, 99, 1360

Rocca, A., Roddier, F., \& Vernin, J. 1974, JOSA, 64, 1000

Roddier, F. 1981, ed. E. Wolf, XIX-edn (Amsterdam: North-Holland), 19, 281

Roddier, F., \& Roddier, C. 1978, in Proc. IAU Coll., 50, ed. J. Davis, \& W. J. Tango, Proc. ESO Conf., 32/1

Sarazin, M., \& Roddier, F. 1990, A\&A, 277, 294

Tatarski, V. I. 1961, Dover, New-York

Tokovinin 2002, PASP, 114:11561166

Tokovinin, A., \& Kornilov, V. 2007, MNRAS, 381, 1179

Tokovinin, A., Kornilov, V., Shatsky, N., \& Voziakova, O. 2003, MNRAS, 343, 891

Vernin, J., \& Muñoz-Tuñón, C. 1994, A\&A, 284, 311

Ziad, A., Conan, R., Tokovinin, A., Martin, F., \& Borgnino, J. 2000, Appl. Opt., 39,30 